\title{
Nine years experiences of solid organ xenotransplantation
}

\author{
Hye Sun Shin ${ }^{1}$, Ik Jin Yun ${ }^{1}$, Hyun Keun Chee ${ }^{2}$, Jun Seok Kim² ${ }^{2}$ Jung Hwan Park ${ }^{3}$, Hyun Suk Yang ${ }^{4}$, Wan Seop Kim ${ }^{5}$, \\ Ki Cheul Shin ${ }^{6}$, Kyoung Sik Park ${ }^{1}$, Keon Bong $\mathrm{Oh}^{7}$
}

\footnotetext{
${ }^{1}$ Department of Surgery, Konkuk University Medical Center, Seoul, Korea

${ }^{2}$ Department of Thoracic and Cardiovascular Surgery, Konkuk University Medical Center, Seoul, Korea

${ }^{3}$ Division of Nephrology, Department of Internal Medicine, Konkuk University Medical Center, Seoul, Korea

${ }^{4}$ Division of Cardiology, Department of Internal Medicine, Konkuk University Medical Center, Seoul, Korea

${ }^{5}$ Department of Pathology, Konkuk University Medical Center, Seoul, Korea

${ }^{6}$ Department of Ophthalmology, Konkuk University Medical Center, Seoul, Korea

${ }^{7}$ Animal Biotechnology Division, National Institute of Animal Science, Wanju, Korea
}

Background: There are far more patients awaiting transplantation than there are organ donors, the problem of organ scarcity has become more profound. One promising alternative could be xenograft using pig organ. There are very few teams in Korea that actively work on xenotransplantation studies, although our team has been conducting xenotransplantation research since 2011.

Methods: Since 2011, with state research funding, our team has been performing solid organ transplantation and partial corneal transplantation preclinical studies using transgenic pigs and non-human primates (NHP). From 2011 to 2020, 24 cases of heart transplantations, 13 cases of kidney transplantations and 10 cases of corneal transplantations were conducted. The transgenic pigs (GTKO) have been used to prevent hyperacute rejection. And we are using genetically engineered pigs with different genetic backgrounds such as CD46, CD73, CD39, and CD55. Immunosuppressive therapy is based on anti-CD154 and appropriately mixed with anti-thymocyte globulin, rituximab, tacrolimus, mycophenolate mofetil rapamycin, and steroid. In the case of corneal transplantation, minimal immunosuppression was applied for partial lamellar corneal transplantation.

Results: Long-term survival records are 60 days for the heart and 84 days for the kidney. The partial lamellar corneal transplantation has the longest record for survival which is more than 3 years that is currently continuing without rejection. And the average survival record for the heart is 17 days, 19.5 days for the kidney and 260 days for the cornea. Since GTKO pigs were used, no hyper-acute rejection has been observed.

Conclusions: Overall, our achievements and experience will be able to help solve the problem of organ shortage. Although the findings are not yet adequate for clinical trials and are relatively poor, further research practices and studies are thought to be achieve better outcomes. Donor genetic backgrounds and combination of immune suppressants increased the graft survival and partial lamellar corneal transplantation could be able to achieve better results.

Corresponding author: Ik Jin Yun

E-mail: ijyun@kuh.ac.kr

(c) The Korean Society for Transplantation

This is an Open Access article distributed under the terms of the Creative Commons Attribution Non-Commercial License (http://creativecommons.org/licenses/by-nc/4.0/) which permits unrestricted non-commercial use, distribution, and reproduction in any medium, provided the original work is properly cited. 